\title{
Long Non-Coding RNA BCAR4 Promotes Growth, Invasion and Tumorigenicity by Targeting miR-2276 to Upregulate MMP7 Expression in Glioma
}

This article was published in the following Dove Press journal: OncoTargets and Therapy

\section{Zhifeng Wang \\ Longlong Wang \\ Zan Liang \\ Yanguo $\mathrm{Xi}$}

Department of Neurosurgery, Cangzhou Central Hospital, Cangzhou, Hebei Province, People's Republic of China
Correspondence: Zhifeng Wang; Yanguo $\mathrm{Xi}$

Department of Neurosurgery, Cangzhou Central Hospital, No. 16 Xinhuaxi Road, Cangzhou City, Hebei 061000, People's

Republic of China

Tel +863172075627

Email zhifengwang_czns@aliyun.com
Objective: Long non-coding RNA breast cancer anti-estrogen resistance 4 (BCAR4) has been recognized as a proto-oncogene in various malignancies. It has been reported to be highly expressed and promote cell proliferation in glioma. However, its additional roles in gliomagenesis remain largely unclear. This research intends to investigate the impact and internal molecular mechanism of BCAR4 on glioma cell growth, invasion and tumorigenesis. Methods: BCAR4 expression was examined by qPCR in 30 cases of graded glioma specimens and 7 glioblastoma (GBM) cell lines compared with respective controls. Its potential prognostic value was evaluated by Kaplan-Meier survival analysis. The biological roles of BCAR4 in gliomagenesis were verified by CCK-8, transwell and intracranial xenograft assays successively. qPCR and RNA pull-down assays were applied to study the relationship between BCAR4 and miR-2276. Then, qPCR, Western blot and luciferase reporter assays were used to validate the targeting of matrix metallopeptidase 7 (MMP7) by miR-2276 and the regulation of MMP7 by BCAR4. Finally, MMP7 was restored in BCAR4-silenced GBM cells and the rescue effects were determined by CCK- 8 and transwell assays.

Results: BCAR4 expression was increased in glioma tissues and GBM cell lines, and its high expression positively correlated with advanced grades and worse prognosis. Functional assays verified that knockdown of BCAR4-inhibited cell growth and invasion in vitro, and impaired tumor formation in vivo. Mechanistically, we found that BCAR4 could act as a competing endogenous RNA (ceRNA) by targeting miR-2276 to upregulate MMP7 expression. Importantly, MMP7 restoration effectively rescued the inhibitory modulations on GBM cell growth and invasion caused by BCAR4 knockdown.

Conclusion: Our findings identified the essential roles of the BCAR4/miR-2276/MMP7 axis in gliomagenesis and provided novel insights on glioma therapy.

Keywords: glioma, BCAR4, ceRNA, miR-2276, MMP7

\section{Introduction}

Deriving from neuroepithelial, glioma is the most common primary tumor in the central nervous system, and malignant glioma is highly lethal. ${ }^{1}$ Among all the subtypes of gliomas, glioblastoma (GBM) belongs to the World Health Organization (WHO) grade IV gliomas, and is easy to relapse due to its infiltration nature which leads to complete resection almost impossible. ${ }^{2,3}$ The highly invasive nature also largely contributes to the poor prognosis of GBM patients. ${ }^{4}$ In spite of the progress achieved in surgical, chemotherapeutic and radio-therapeutic treatment, the overall clinical outcome of GBM patients remains far from satisfactory. ${ }^{4}$ Therefore, exploring more effective 
prognostic biomarkers and therapeutic targets especially to overcome the invasion function are urgently needed to improve the current situation.

Long non-coding RNAs (lncRNAs) are a group of RNAs with very limited protein-coding potential containing more than 200 nucleotides. $^{5}$ As the research goes on, lncRNAs are recognized as important regulators in cancer development by impacting on various cellular processes, such as cell differentiation, proliferation, apoptosis and metastasis. ${ }^{6-8}$ They function as either tumor suppressors or oncogenes in specific cancers. ${ }^{8}$ Due to their versatile biological activities and tumor-restricted expression patterns, lncRNAs are deemed as promising biomarkers and therapeutic target in cancers, including in glioma. ${ }^{9-11}$ For instance, scholars demonstrated that expressions of lncRNAs like HOTAIR, HOXA11-AS and XIST are positively correlated with the malignancy of glioma, while lncRNAs like HOTTIP and GAS5 exhibit the negative correlation. In addition, the tumor-suppressing GAS5 indicates favorable prognosis, whereas oncogenic HOTAIR, HOXA11-AS, H19 and XIST indicate poor prognosis of glioma patients. ${ }^{11}$ Mechanistically, emerging evidences have supported that lncRNAs are implicated in gene expression regulation by acting as competing endogenous RNAs (ceRNAs) to competitively binding to microRNAs (miRNAs). ${ }^{12-14}$ MiRNAs are another group of non-coding RNAs with approximately 21 nucleotides in length and suppress target gene expression via base-pairing to the $3^{\prime}$ untranslated region (3'-UTR) of the target mRNAs. ${ }^{15}$ The interactions among lncRNAs, miRNAs, and the target mRNAs of miRNAs, constitute the ceRNA network, and play critical roles in the development and progression of human cancers. ${ }^{16,17}$

LncRNA breast cancer anti-estrogen resistance 4 (BCAR4) has been reported to be abnormally overexpressed in several carcinomas, including in glioma. ${ }^{18-21}$ The known modulation of BCAR4 in gliomagenesis is that it promotes cell proliferation. ${ }^{21}$ However, BCAR4 also possesses other oncogenic activities such as facilitating cancer cell migration, invasion and epithelial-mesenchymal transition (EMT) in other types of cancers. ${ }^{22,23}$ Therefore, its roles in gliomas deserve more attention.

In this study, we checked the expression and prognostic significance of BCAR4 in gliomas, and verified its biological functions in cell growth, invasion and tumorigenicity by employing both in vitro and in vivo assays. Bioinformatics screening predicted that miR-2276 might interact with BCAR4, and MMP7 was one of the targets of miR-2276. Substantial experiments were performed to validate the regulatory ceRNA network of BCAR4/miR-2276/MMP7 in GBM cells. Finally, MMP7 was restored in BCAR4-silenced GBM cells and the rescue effects on cell growth and invasion were examined. Our study therefore demonstrated a novel BCAR4/miR-2276/MMP7 signaling axis contributing to gliomagenesis and provided valuable insights in discovering potential biomarkers and therapeutic targets for this disease.

\section{Materials and Methods}

\section{Patients and Tissues}

Ten non-tumoral brain tissues (from cerebral trauma patients without neoplasm) and 30 glioma tissues (from glioma patients) were collected from Cangzhou Central Hospital from Jan 2017 to Dec 2018. After surgical excision, all samples were histopathologically diagnosed by pathologists and classified according to WHO classification. All patients did not receive chemotherapy or radiotherapy prior to sample collection. Tissue samples were frozen immediately in liquid nitrogen and stored at $-80^{\circ} \mathrm{C}$ for RNA extraction. The basal clinical features of the 30 glioma patients enrolled in this study are listed in Table 1. Written informed consent had been obtained from all the included patients and the experiment was conducted in accordance with the Declaration of Helsinki. This study was also approved by the Ethics Committee of Cangzhou Central Hospital and the written informed consents were collected from all patients.

\section{Cell Culture and Transfection}

GBM cell lines (U87, U251, U343, LN229, LN308, A172 and SNB19) and normal human astrocytes (NHA) were from the Cell Bank of Institute of Biochemistry and Cell Biology of the Chinese Academy of Sciences (Shanghai, China). DMEM medium (Thermo Fisher Scientific, Waltham, MA, USA) supplemented with $10 \%$ fetal bovine serum (Thermo Fisher Scientific, Waltham, MA, USA), $100 \mathrm{U} / \mathrm{mL}$ penicillin, and $100 \mathrm{mg} / \mathrm{mL}$ streptomycin (Thermo Fisher Scientific, Waltham, MA, USA) was used to culture cells at $37^{\circ} \mathrm{C}$ with $5 \% \mathrm{CO}_{2}$. For transfections, cells were cultured using complete medium without antibiotics at least $24 \mathrm{hrs}$ prior to transfection. Cells were washed twice by PBS and transiently transfected with siRNAs, miRNAs or plasmids by using Lipofectamine ${ }^{\mathrm{TM}}$ 2000 Transfection Reagent (Thermo Fisher Scientific, Waltham, MA, USA) as per the manufacturer's instructions.

\section{SiRNAs, miRNAs, Lentivirus and Plasmids}

BCAR4 siRNAs, non-labeled or biotin-labeled miR-2276$3 \mathrm{p}$ mimics and negative control oligos were all purchased 
Table I The Clinical Features of the 30 Glioma Specimens Enrolled in This Study

\begin{tabular}{|c|c|c|c|c|}
\hline \multirow[t]{2}{*}{ Feature } & \multicolumn{4}{|c|}{ WHO Grade } \\
\hline & $\begin{array}{l}\text { I } \\
(n=2)\end{array}$ & $\begin{array}{l}\text { II } \\
(n=6)\end{array}$ & $\begin{array}{l}\text { III } \\
(n=10)\end{array}$ & $\begin{array}{l}\text { IV } \\
(n=\mid 2)\end{array}$ \\
\hline \multicolumn{5}{|l|}{ Gender } \\
\hline Male & 2 & 3 & 6 & 8 \\
\hline Female & 0 & 3 & 4 & 4 \\
\hline Age (year, & 27.50 & 46.17 & 45.10 & 55.42 \\
\hline mean $\pm S D$ ) & \pm 3.54 & \pm 8.47 & \pm 8.77 & \pm 7.63 \\
\hline Age $<50$ & 2 & 4 & 7 & 4 \\
\hline Age $\geq 50$ & 0 & 2 & 3 & 8 \\
\hline \multicolumn{5}{|l|}{ Predominant side } \\
\hline Left & 1 & 3 & 4 & 5 \\
\hline Right & 1 & 2 & 5 & 4 \\
\hline Middle & 0 & I & I & 3 \\
\hline \multicolumn{5}{|l|}{ Predominant } \\
\hline \multicolumn{5}{|l|}{ location } \\
\hline Frontal lobe & I & 4 & 6 & 8 \\
\hline Temporal lobe & I & I & 2 & 2 \\
\hline Parietal lobe & 0 & 0 & 2 & 2 \\
\hline Occipital lobe & 0 & I & 0 & 0 \\
\hline \multicolumn{5}{|l|}{ KPS score } \\
\hline$<90$ & 0 & 3 & 7 & 8 \\
\hline$\geq 90$ & 2 & 3 & 3 & 4 \\
\hline
\end{tabular}

Abbreviation: KPS, karnofsky performance score.

from GenePharma (Shanghai, China). Lentivirus containing BCAR4 shRNA or negative control lentivirus were purchased from GenePharma (Shanghai, China). U87 cells were infected with lentivirus at an MOI of 10 using $10 \mu \mathrm{g} /$ $\mathrm{mL}$ polybrene (Sigma, St. Louis, MO, USA). The wild type or mutated-type sequence of 3'-UTR region of MMP7 mRNA was cloned into pmirGLO vector (Promega, Madison, WI, USA). The wild-type sequence of MMP7 was cloned into pcDNA3.1 vector (Thermo Fisher Scientific, Waltham, MA, USA).

\section{Quantitative Real-Time PCR (qPCR)}

Total RNA was extracted from tissue samples or cells by using TRIzol reagent (Thermo Fisher Scientific, Waltham, MA, USA) as per the manufacturer's instructions. $1 \mu \mathrm{g}$ of RNA were reverse transcribed to detect miR-2276-3p and U6B expression level using specific primer kit (RiboBio, Guangzhou, China). $2 \mu \mathrm{g}$ of RNA were reverse transcribed to detect BCAR4, MMP7 and $\beta$-actin expression level using general reverse transcription kit (Promega, Madison, WI,
USA). qPCR was performed using SYBR Green PCR Master Mix (Takara, Otsu, Japan) on the Applied Biosystems 7500 Real-Time PCR System (Thermo Fisher Scientific, Waltham, MA, USA). The level of miR-2276-3p was normalized to that of U6B. The expressions of BCAR4 and MMP7 were standardized to the control values of $\beta$ actin. The relative expression of target genes was calculated with the $2^{-\Delta \Delta \mathrm{Ct}}$ methods.

\section{CCK-8 Assay}

Transfected U87 and LN229 cells were seeded at a density of $5 \times 10^{3}$ cells per well in a 96-well plate containing 100 $\mu \mathrm{L}$ DMEM complete medium. Cell Counting Kit- 8 (Dojindo, Tokyo, Japan) reagent was added at Days 1-5 after seeding (Day 0) and incubated at $37^{\circ} \mathrm{C}$ for $2 \mathrm{hrs}$. The data of optical density of OD450 were measured by a microplate reader (Molecular Device, LLC, California, USA). Each experiment was performed three times with six replicates.

\section{Transwell Assay}

Transwell assay was performed to detect the cell invasion ability. Invasion assay was carried out by using transwell chambers (Corning, New York, USA). $3 \times 10^{3}$ transfected U87 or LN229 cells seeded into the $1 \mathrm{mg} / \mathrm{mL}$ matrigelcoated upper chamber were cultured at $70 \%$ confluence in $500 \mu \mathrm{L}$ FBS free medium. Medium with $10 \%$ FBS (200 $\mu \mathrm{L})$ was added to the lower chamber. After $24 \mathrm{hrs}$ culturing, the invaded cells were fixed with $4 \%$ paraformaldehyde, followed by staining with $0.25 \%$ crystal violet. Evaluation of cell invasion ability was performed by counting invaded cells under an inverted microscope (Olympus, 100×), and five different microscopic views were selected randomly for analysis.

\section{Western Blot}

Total protein was extracted using RIPA lysis buffer (Beyotime Biotechnology, Nanjing, China) as per the manufacturer's instructions. Protein samples were separated by 9\% SDS-PAGE and then transferred onto nitrocellulose membranes (EMD Millipore, Billerica, USA). After incubated and blocked in the 5\% skim milk in PBST buffer for $1 \mathrm{hr}$ at room temperature, the membranes were incubated with anti-MMP7 antibody (Cell Signaling Technology, Danvers, MA, USA; $1: 1000)$ and anti- $\beta$-actin antibody (Sigma, St. Louis, MO, USA; $1: 8000$ ) at $4^{\circ} \mathrm{C}$ overnight, respectively. On the following day, the membranes were incubated with HPR-conjugated secondary antibodies at 
room temperature for $1 \mathrm{hrs}$. The blots were measured using an enhanced chemiluminescence detection system (Pierce, Rockford, IL, USA).

\section{Dual-Luciferase Reporter Assays}

U87 and LN229 cells were seeded in 24-well plates at a density of $1.5 \times 10^{5}$ cells per well 24 hrs before transfection. Cells were transfected with MMP7-3'-UTR-WT or MMP73'-UTR-MT, followed by transfection with miR-2276 mimics or negative control oligos. $48 \mathrm{hrs}$ later, cells were harvested, and firefly and renilla luciferase activities were measured using a dual-luciferase reporter assay system (Promega, Madison, WI, USA) as per the manufacturer's instructions.

\section{RNA Pull-Down Assay with Biotinylated miR-2276}

U87 and LN229 cells were transfected with biotin-labeled wild-type miR-2276-3p (Bio-miR-2276-WT), mutatedtype miR-2276-3p (Bio-miR-2276-MT) or antagonistic miR-2276-3p probe (Bio-miR-NC). 48 hrs after transfection, cells were harvested and incubated with a specific lysate buffer (Ambion, Austin, TX, USA) for 10 mins. After that, the cell lysates were mixed with M-280 streptavidin magnetic beads (Sigma, St. Louis, MO, USA) for 3 hrs at $4^{\circ} \mathrm{C}$. The pull-down products were subjected to qPCR for BCAR4 expression.

\section{Intracranial Xenograft Assay in Nude Mice}

The constructed U87 sub-cell lines expressing luciferase and indicated shRNAs (NC-sh and BCAR4-sh2) were transplanted intracranially $\left(5 \times 10^{4}\right.$ cells per mouse) into the brains of 4-week old female BALB/C athymic nude mice using cranial guide screws. The intracranial tumor volume was detected by bioluminescence imaging every week after transplantation (at days 7, 14, 21). All animal experimental procedures were approved by the Institutional Animal Care and Use Committee of Cangzhou Central Hospital. All experiments were performed following guidelines and regulations of Committee of Cangzhou Central Hospital.

\section{Statistical Analysis}

Data were presented as mean \pm SD (standard deviation). Significant differences were determined by Student's $t$-test or one-way ANOVA by using GraphPad Prism v6 software (GraphPad Software, Inc., San Diego, CA).
Survival analysis was performed by Kaplan-Meier method and log-rank test. Pearson correlation test was performed to confirm the correlation between BCAR4 and miR-2276$3 p$ expressions. A P value of $<0.05$ was considered statistically significant.

\section{Results \\ Overexpression of BCAR4 in Gliomas and Its Prognostic Significance}

To validate the expression of BCAR4 in gliomas, we firstly examined its expression levels in 30 cases of graded glioma specimens and 10 non-tumoral brain tissues by qPCR. We found that BCAR4 was overexpressed in the 30 gliomas compared with normal brains (Figure 1A). Importantly, among these 30 cases of gliomas, BCAR4 expression was higher in advanced grades (WHO grades III-IV) than in lower grades (WHO grades I-II; Figure 1B). Besides, grade IV gliomas expressed higher BCAR4 than grade III gliomas (Figure 1B). In other words, its expression positively correlated with glioma grades. We also checked its expression in GBM cell lines and the normal human astrocytes (NHA). The result came out the same. BCAR4 expression was increased in the tested GBM cell lines (Figure 1C). Therefore, the expression detection confirmed the overexpression of BCAR4 in gliomas, which was in consistence with the previous finding by Wei et al. ${ }^{21}$ We then analyzed its prognostic significance by employing Kaplan-Meier survival analysis. As shown in Figure 1D, patients with higher BCAR4 levels showed a significant poorer progression-free survival (PFS) and overall survival (OS) rate. These initial results implied that BCAR4 might be crucial for gliomagenesis.

\section{BCAR4 Knockdown Impairs GBM Cell Growth, Invasion and Tumorigenicity}

Then, we systematically explored the biological functions of BCAR4 in gliomagenesis. Two different small interfering RNAs (siRNAs) targeting BCAR4 were used to transiently knockdown BCAR4 in GBM cells. U87 and LN229 cell lines were selected since they contained relatively higher levels of BCAR4 (Figure 1C). The inhibition efficiency was checked by qPCR (Figure 2A). CCK-8 assay verified that BCAR4 knockdown led to growth inhibition in both U87 and LN229 cell lines (Figure 2B). Transwell assay demonstrated that BCAR4 knockdown prominently suppressed GBM cell invasion (Figure 2C). To further make sure whether BCAR4 was critical for 
A

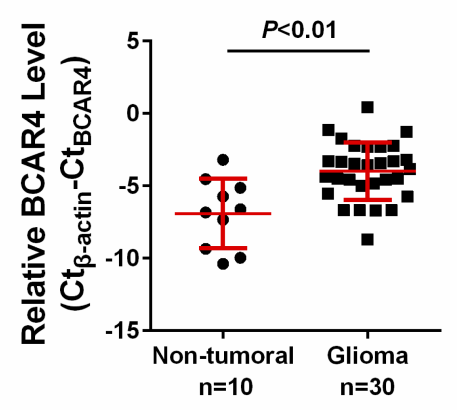

C

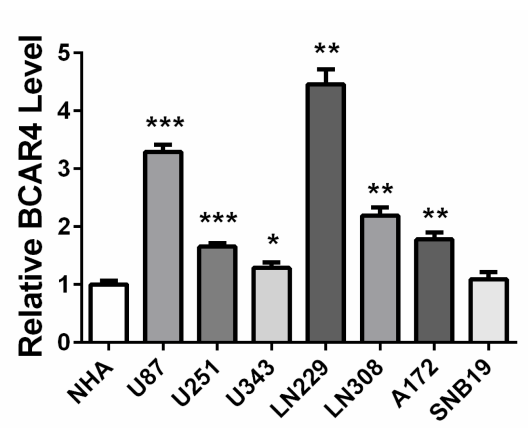

B
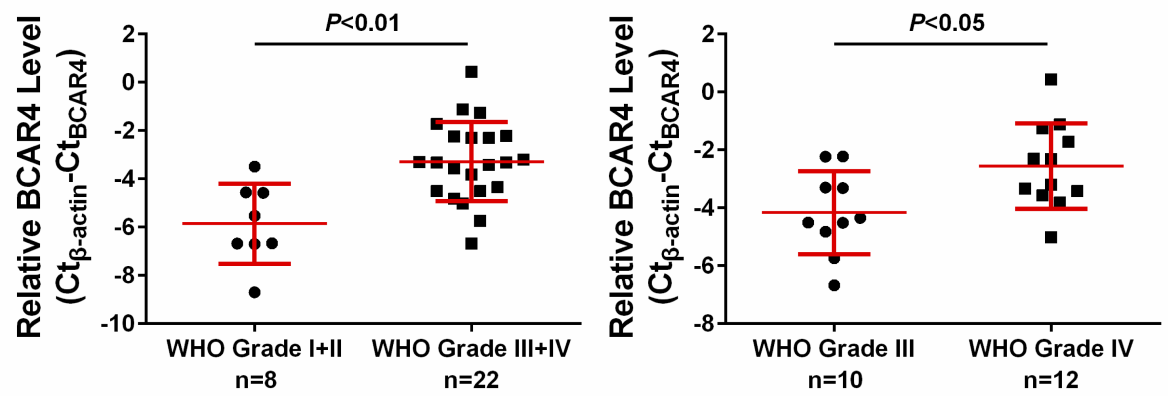

D
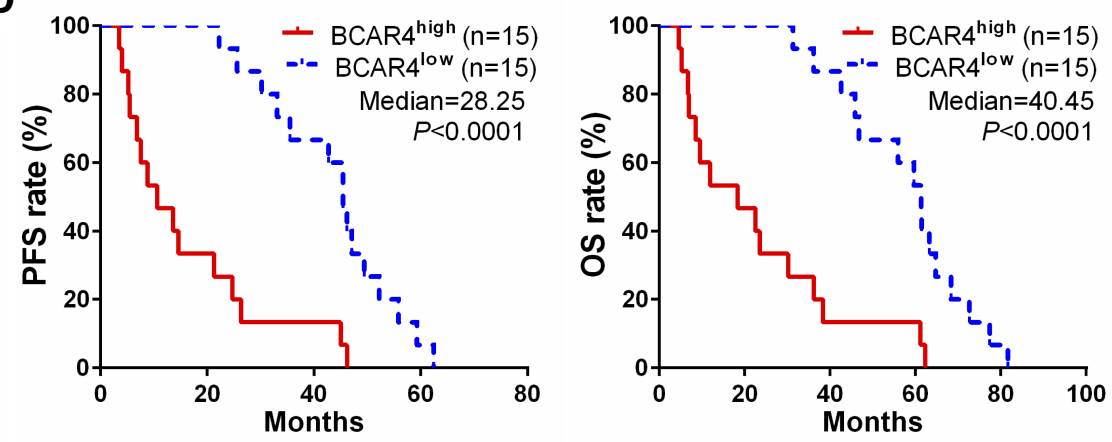

Figure I Long non-coding RNA BCAR4 is overexpressed in glioma. (A) The expression difference of BCAR4 was compared in non-tumoral brain tissues ( $\mathrm{n}=\mathrm{I0}$ ) and graded glioma specimens $(n=30)$. (B) Left: The difference of BCAR4 was compared in gliomas at lower grades (WHO grades I-II, $n=8)$ and at advanced grades (WHO grades III-IV, $n=22)$. Right: The difference of BCAR4 was compared in gliomas at grade III $(n=10)$ and at grade IV $(n=I 2)$. (C) Relative expression of BCAR4 in GBM cell lines (U87, U25I, U343, LN229, LN308, AI72 and SNB19) and normal human astrocytes (NHA). (D) The patients were divided into two subgroups based on the median level of BCAR4. Glioma patients with higher BCAR4 level $(n=15)$ had a significantly poorer progression-free survival (PFS, left panel) and overall survival (OS, right panel) compared to lower BCAR4 expressing cases $(n=15)$. $* P<0.05$, $* * P<0.01$, and $* * * P<0.001$.

gliomagenesis in vivo, we constructed the stable BCAR4 knockdown U87 sub-cell line by utilizing its shRNA expressing lentivirus (Figure 2D). The following intracranial xenograft experiment showed that stable BCAR4 knockdown significantly inhibited tumor formation (Figure 2E). Therefore, our functional assays indicated the oncogenic roles of BCAR4 in gliomagenesis.

\section{BCAR4 Modulates miR-2276/MMP7 Pathway in GBM Cells}

To investigate the molecular mechanism of BCAR4 in promoting gliomagenesis, we screened possible miRNA regulators of BCAR4 by using DIANA-LncBase v2 ${ }^{24}$ and found that miR-2276 was a potential target of BCAR4 (Figure 3A). qPCR showed that BCAR4 knockdown led to increased expression of miR-2276 in both U87 and LN229 cell lines (Figure 3B). In addition, in the abovementioned 30 gliomas, BCAR4 expression was inversely correlated with miR-2276 (Figure 3C), further demonstrating the negative regulation between BCAR4 and miR-2276. RNA pull-down confirmed the interaction between BCAR4 and miR-2276 (Figure 3A and D).
Then, we predicted the mRNA targets of miR-2276 by using TargetScan Release 7.2 and among all the targets of miR-2276, we mainly focused on matrix metallopeptidase 7 (MMP7; Figure 4A). As is known, MMP7 is implicated in glioma cell proliferation and invasion and closely associated with gliomagenesis. ${ }^{25}$ qPCR and Western blot assays revealed that miR-2276 mimics transfection led to reduced expression of MMP7 mRNA and protein in both U87 and LN229 cell lines (Figure 4B and C). Luciferase reporter assay also indicated that MMP7 was a direct target of miR2276 in GBM cells (Figure 4A and D). Intriguingly, BCAR4 knockdown obviously inhibited the expression of MMP7 in both U87 and LN229 cell lines (Figure 4E and F). Therefore, our results identified that BCAR4 might act as a competing endogenous RNA (ceRNA) by interacting with miR-2276 to upregulate MMP7 expression in glioma cells.

\section{MMP7 Restoration Rescues the Tumor Suppressing Effects of BCAR4 Knockdown}

As we have found that BCAR4 was crucial for GBM cell growth, invasion and tumorigenicity, and it positively 

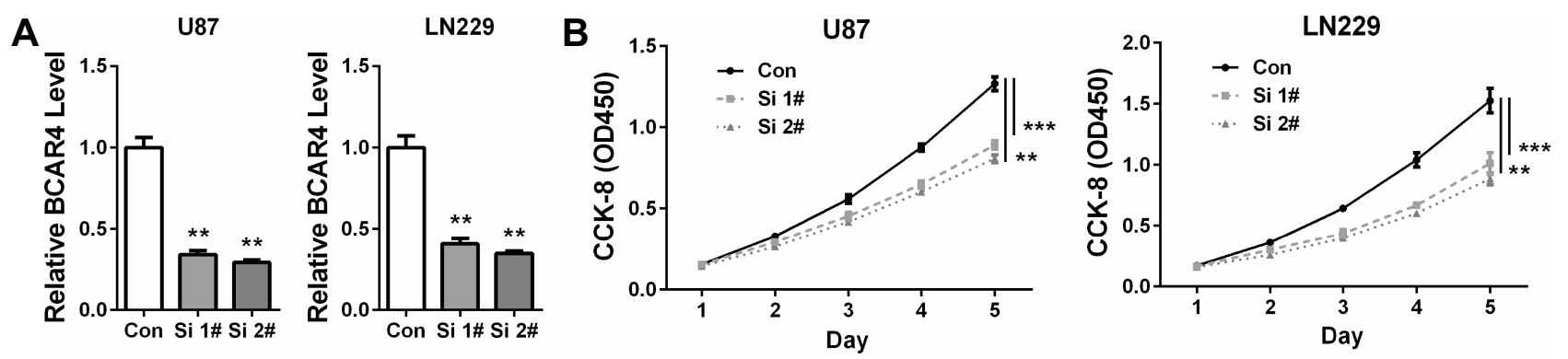

C
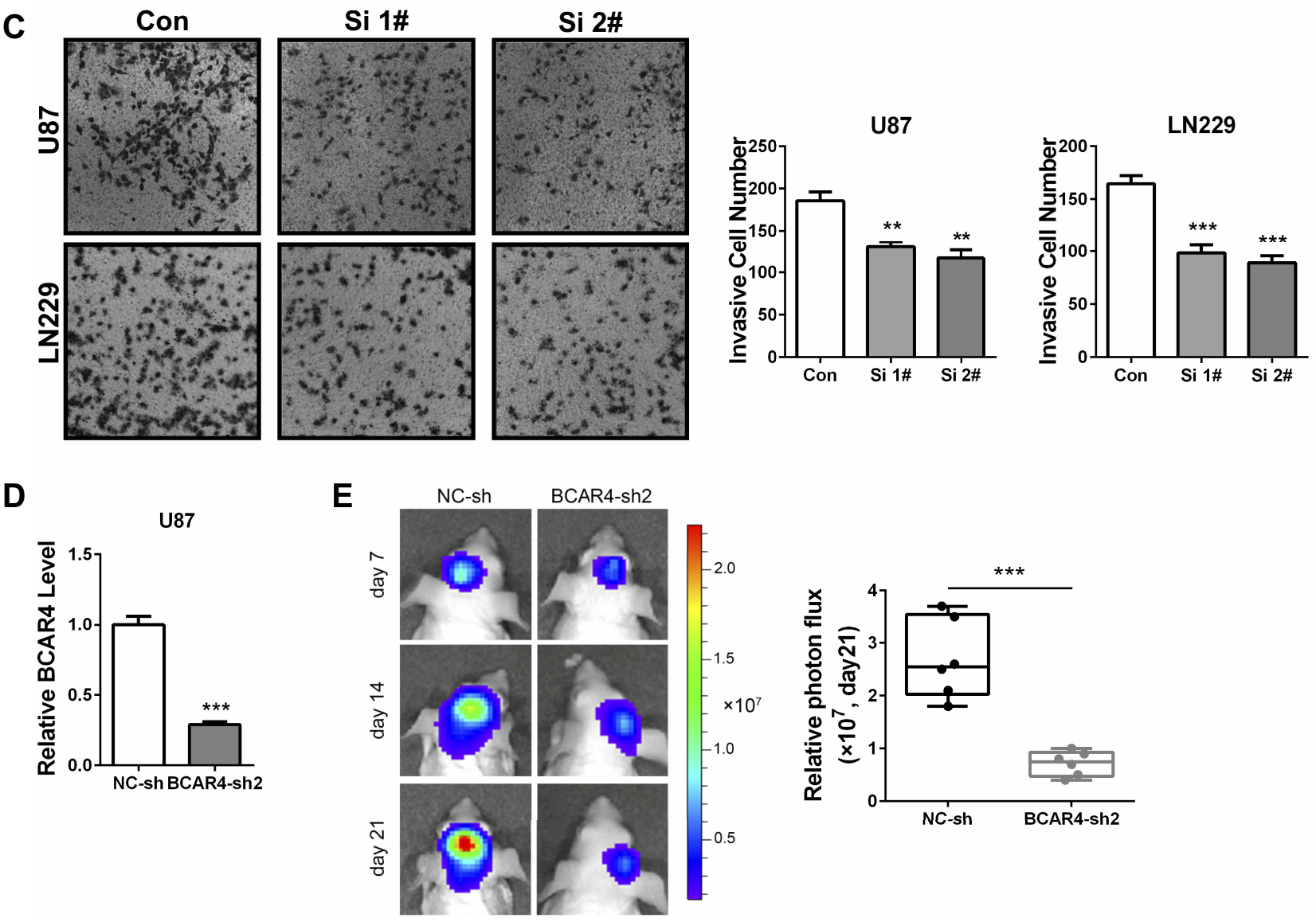

Figure 2 BCAR4 knockdown impairs the cell growth, invasion and tumorigenicity of glioma cells. (A) U87 and LN229 cells that transfected with two different siRNAs against BCAR4 (Si I\# and Si 2\#) or negative control siRNA (Con) were subjected to qPCR for BCAR4 expression. (B) CCK-8 assay demonstrated that BCAR4 knockdown impaired the cell growth of glioma cells. (C) Transwell assay indicated that the invasion abilities of U87 and LN229 cells were weakened by BCAR4 knockdown. (D) U87 cells that were infected with BCAR4 shRNA expressing lentivirus (BCAR4-sh2) or negative control lentivirus (NC-sh) were subjected to qPCR for BCAR4 expression. (E) U87 sub-cell line bearing control shRNA (NC-sh) or shRNA against BCAR4 (BCAR4-sh2) were inoculated orthotopically into nude mice ( $n=5$ for each group). Bioluminescence images of representative mice are shown (left panel). Representative tumor volumes were revealed by the photon flux at day $2 \mathrm{I}$ after implantation (right panel). $* * \mathrm{P}<0.0 \mathrm{I}$ and $* * * \mathrm{P}<0.00 \mathrm{I}$.

regulated MMP7 expression via targeting miR-2276, we finally intend to verify whether MMP7 could be the downstream effector of BCAR4 in promoting gliomagenesis. MMP7 was restored in BCAR4-silenced U87 and LN229 cells (Figure 5A and B). CCK-8 and transwell assays indicated that MMP7 overexpression resulted in enhanced GBM cell growth and invasion, and its restoration in BCAR4silenced cells effectively abolished the inhibition of cell growth and invasion caused by BCAR4 knockdown in both
U87 and LN229 cells (Figure 5C and D). Altogether, our study uncovered that MMP7 was a critical effector in mediating the oncogenic roles of BCAR4 in glioma.

\section{Discussion}

The present study described the essential roles of the lncRNA BCAR4 in gliomagenesis. Firstly, BCAR4 was confirmed to be overexpressed in glioma tissues and GBM cell lines, and its higher expression was positively associated with the 
A

miR-2276-3p-WT 3' GGAGCGGAGA-------CUG--------UGAACGUCU 5'

I | I | | I I | | | | | | | |

BCAR4 (78-103) 3' UACGGUUACAGUCGACGAGAACUUGCAGA 5'

miR-2276-3p-MT 3' GGAGCGGAGA----.--CUG--.----UCUUGCAGU 5'

B

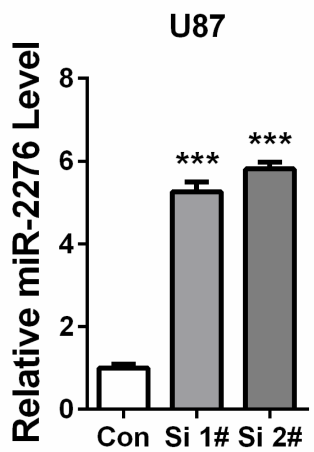

LN229

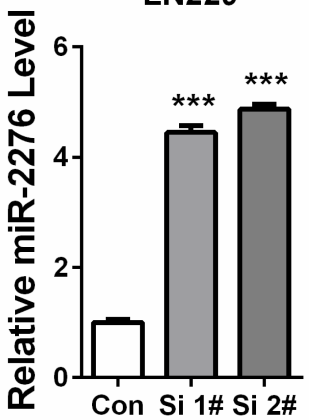

C

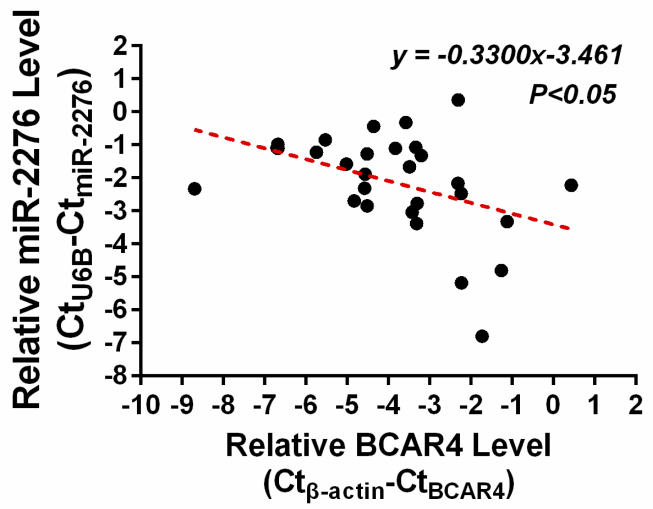

D

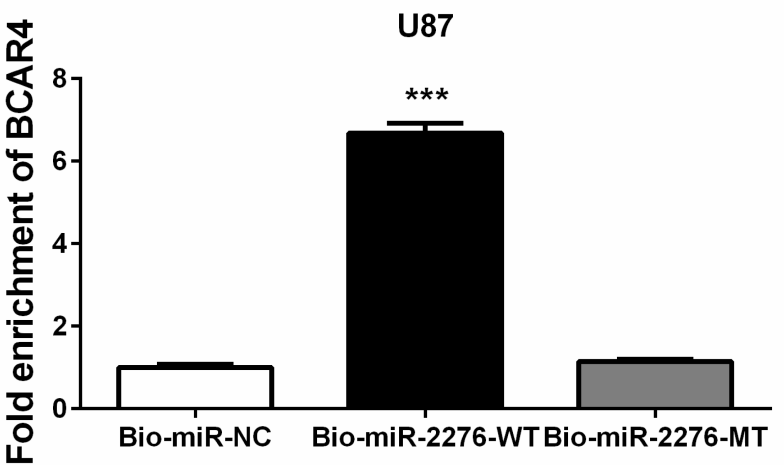

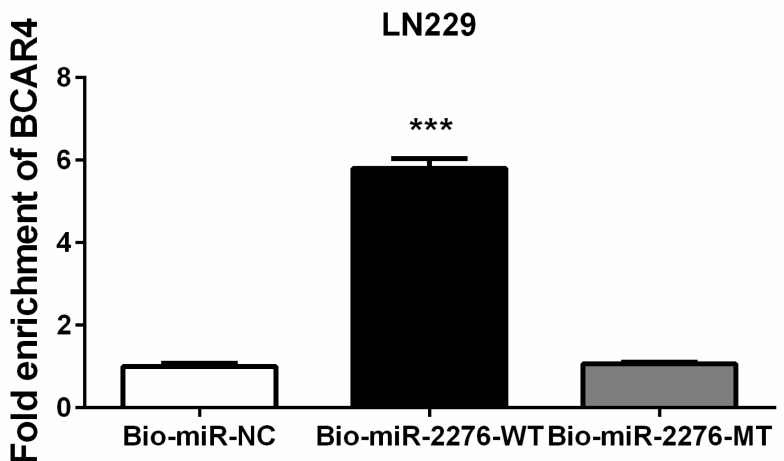

Figure 3 BCAR4 modulates miR-2276 expression in GBM cells. (A) miR-2276-3p wide-type (miR-2276-3p-WT, in green) and the mutated-type (miR-2276-3p-MT, in red) sequences in the IncRNA BCAR4 binding sites (in blue) were shown according to DIANA-LncBase v2. (B) BCAR4 knockdown increased the expression of miR-2276-3p in both U87 and LN229 cells. (C) BCAR4 expression was inversely correlated with miR-2276-3p expression in glioma tissues ( $\mathrm{n}=30$ ). (D) BCAR4 was pulled down by biotinylated wide-type miR-2276-3p (Bio-miR-2276-WT), while there was no interaction between BCAR4 and mutated-type miR-2276-3p (Bio-miR-2276-MT) or biotinlabeled antagonistic miR-2276-3p probe (Bio-miR-NC). $* * * \mathrm{P}<0.00 \mathrm{I}$.

advanced glioma grades and worse prognosis of the patients. In vitro and in vivo loss-of-function assays revealed that BCAR4 knockdown inhibited GBM cell growth, invasion and tumor formation significantly. Importantly, we identified a novel molecular mechanism that BCAR4 acted as a ceRNA by targeting miR-2276 to upregulate MMP7 expression in glioma cells (Figure 6). Finally, functional assays demonstrated that MMP7 played an essential role in the oncogenic roles of BCAR4 in glioma. Taken together, our findings illustrated that the newly disclosed BCAR4/miR-2276/ MMP7 axis-facilitated gliomagenesis and could be developed as a novel therapeutic target for glioma treatment.
Along with the research goes on, lncRNAs are widely recognized as promising diagnostic and prognostic biomarkers in glioma. ${ }^{26}$ For instance, lncRNA HOTAIR was found to be increased in GBM patients' serum and it might be applied as an effective diagnostic and prognostic biomarker for glioma patients. ${ }^{27}$ Herein, we validated that BCAR4 was overexpressed in glioma, keeping in accordance with the previous study by Wei et al. ${ }^{21}$ More importantly, we found that its higher expression positively correlated with advanced glioma grades and worse clinical outcome of the patients. These findings verified the potential clinical significance of BCAR4 in predicting the prognosis of glioma patients. 
A

MMP7-3'-UTR-WT (198-204 ) 5' ...UCUUAUUCAUCUAUA - CUUGCAGU... 3'

hsa-miR-2276-3p

I | I I | | | | | |

3' GGAGCGGAGACUGUGAACGUCU 5'

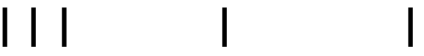

MMP7-3'-UTR-MT (198-204 ) 5' ...UCUUAUUCAUCUAUA - CAACGUGU... 3'

B

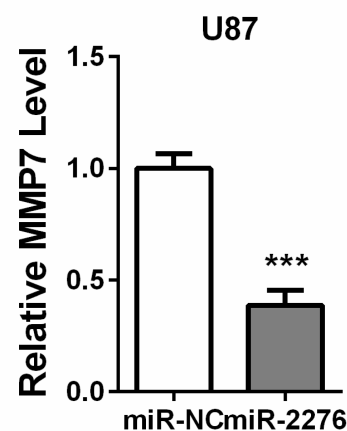

LN229

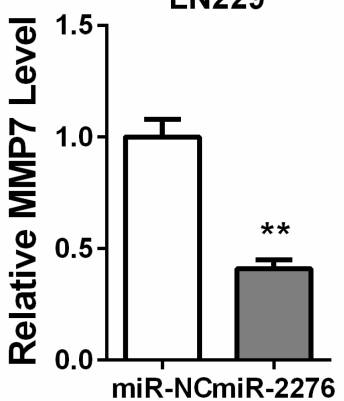

C

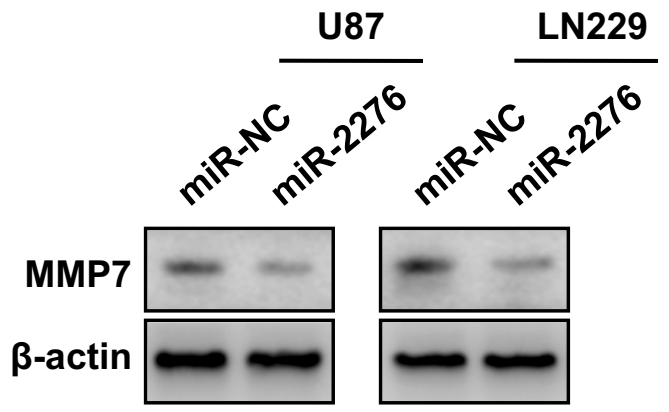

D

U87

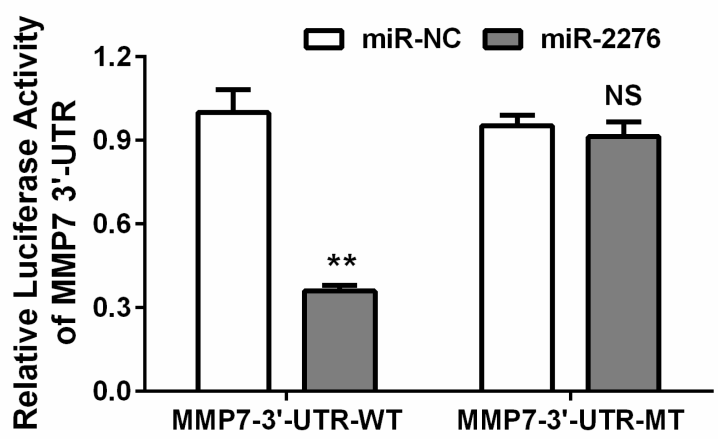

E
LN229

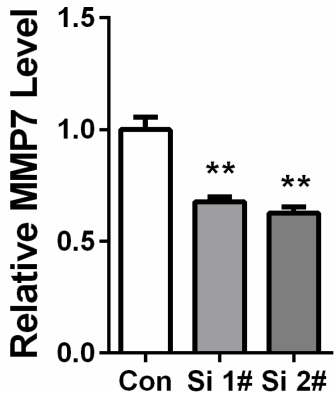

LN229

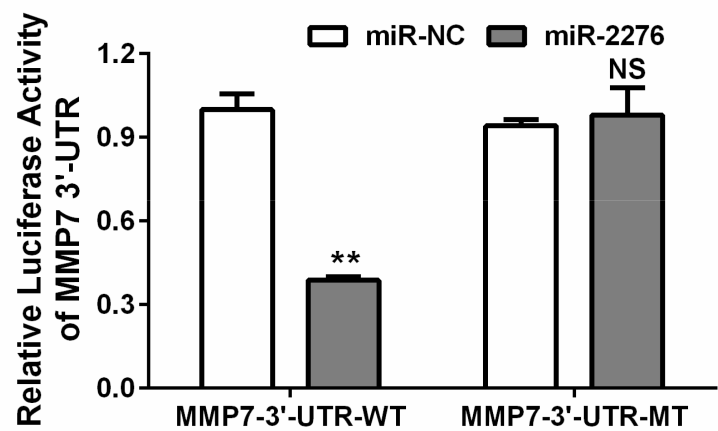

F

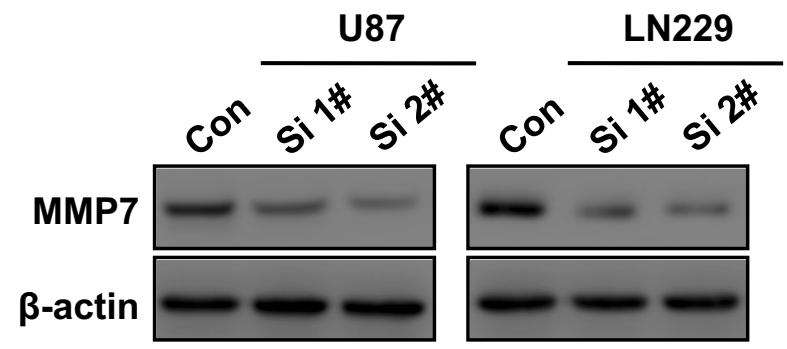

Figure 4 BCAR4 modulates the miR-2276/MMP7 pathway in GBM cells. (A) MMP7 3'-UTR wide-type (MMP7-3'-UTR-WT, in green) and the mutated-type (MMP7-3'-UTRMT, in red) sequences in the miR-2276-3p binding sites (in blue) were shown according to TargetScan Release 7.2. (B) miR-2276 overexpression decreased the mRNA level of MMP7 in both U87 and LN229 cells by qPCR. (C) miR-2276 overexpression decreased the protein expression of MMP7 in both U87 and LN229 cells by Western blot. (D) Luciferase reporter assay indicated that miR-2276-3p overexpression significantly reduced the luciferase activity of MMP7-3'-UTR-WT but not MMP7-3'-UTR-MT in both U87 and LN229 cell. (E) BCAR4 knockdown decreased the mRNA level of MMP7 in both U87 and LN229 cells by qPCR. (F) BCAR4 knockdown decreased the protein expression of MMP7 in both U87 and LN229 cells by Western blot. **P $<0.01$ and ***P $<0.001$. 
A

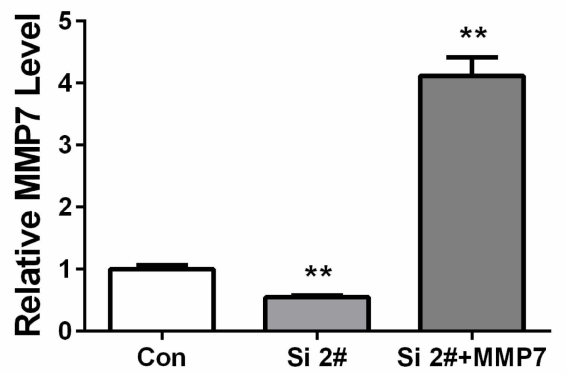

C

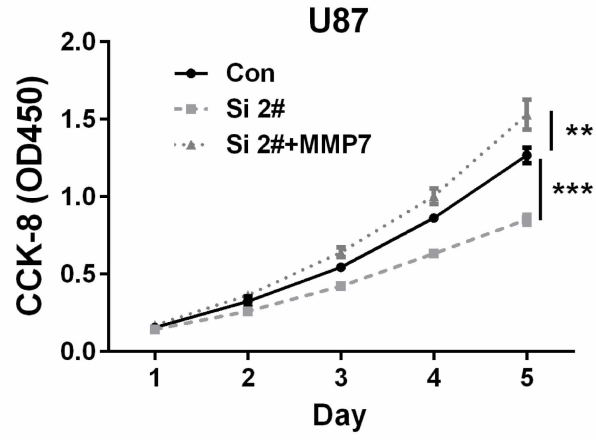

D

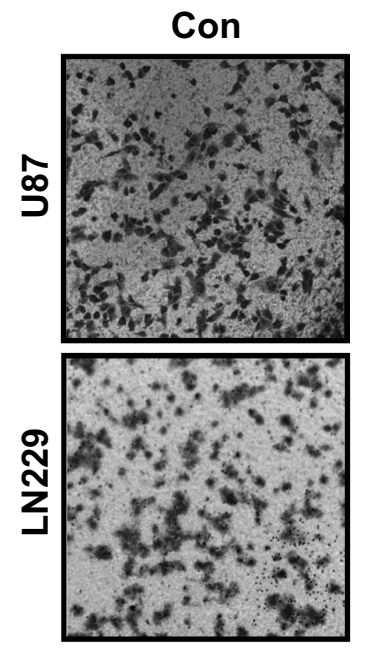

Si 1\#

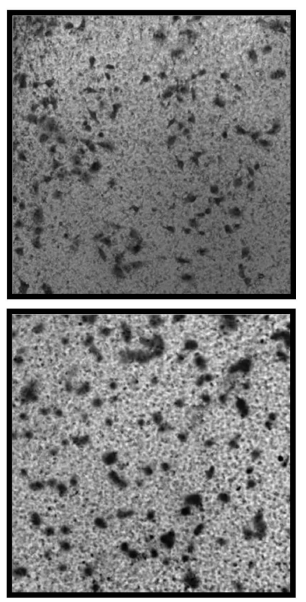

B

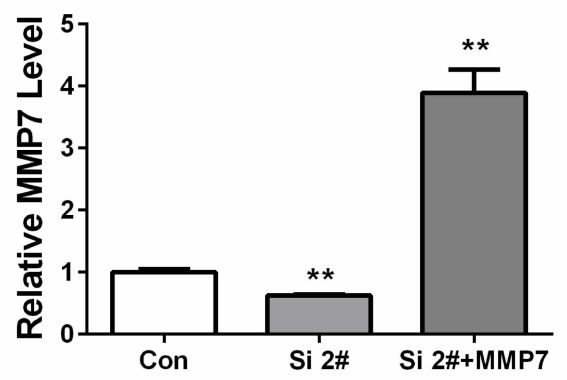

Figure 5 MMP7 restoration rescues the tumor-suppressing effects of BCAR4 knockdown in glioma cells. (A) MMP7 expression was restored by transfecting expression plasmids into BCAR4-silenced U87 cells and qPCR was performed to detect MMP7 expression. (B) MMP7 expression was restored by transfecting expression plasmids into BCAR4-silenced LN229 cells and qPCR was performed to detect MMP7 expression. (C) CCK-8 assay was performed to determine the cell growth in the cells treated as in (A, B). (D) Transwell assay was performed to determine the cell invasion in the cells treated as in (A, B). ${ }^{* * P}<0.01$ and $* * * P<0.001$.

There are also amounting evidences that lncRNA are vital contributors for glioma pathogenesis and progression. ${ }^{26}$ For BCAR4, Wei et al have proved that BCAR4 could promote glioma cell proliferation. ${ }^{21}$ Its biological functions have also been investigated in other types of cancers, including in chondrosarcoma and lung cancer, in which BCAR4 facilitates cancer cell migration, invasion and EMT. ${ }^{22,23}$ In this study, we presented valid evidences to support that BCAR4 facilitated GBM cell growth, invasion and tumorigenicity, fully expanding its oncogenic roles in glioma.
Among the known molecular mechanisms of lncRNAs, ceRNA attracts intensive attention recently. Through targeting miRNAs, lncRNAs could positively regulate the expression of the downstream targets and exert their functions. ${ }^{12-14}$ For BCAR4, it has been demonstrated that it could promote the stemness of colorectal cancer cells through targeting to miR-665/STAT3 signaling. ${ }^{20}$ Herein, we identified BCAR4 as the sponge for miR-2276 in glioma. Up to date, there are no reports regarding the roles of miR-2276 in human cancers. It has merely been mentioned that miR-2276 was decreased 


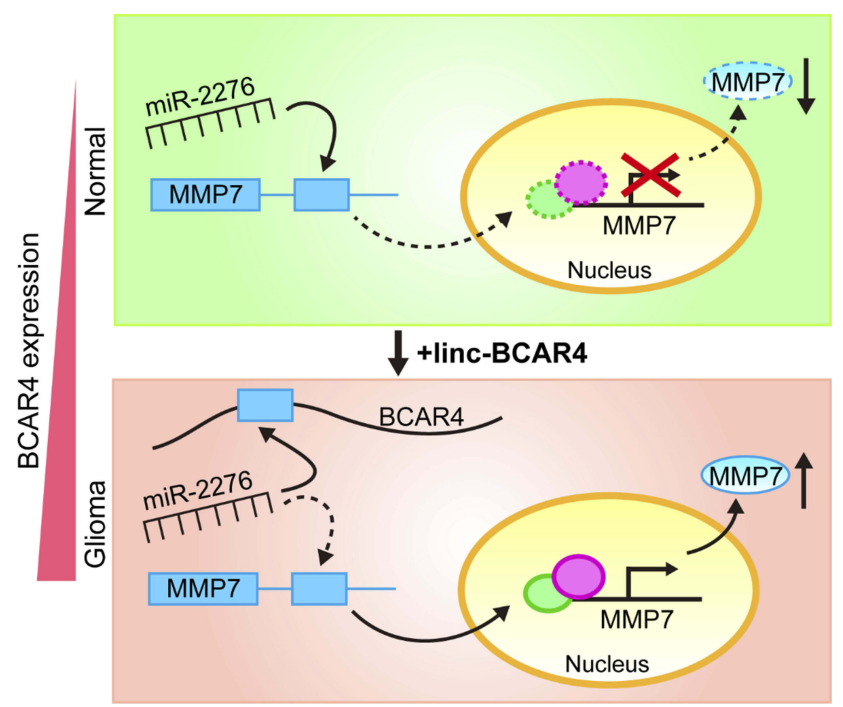

Figure 6 Schematic illustration of the molecular pathway in which BCAR4/miR2276/MMP7 forms the ceRNA relationship in glioma cells.

in breast cancer samples, especially in those at higher grades and with lymph node metastasis. ${ }^{28}$ Our results showed that miR-2276 could be induced by BCAR4 knockdown, and it was downregulated in gliomas and exhibiting inverse correlation with BCAR4 expression. Subsequently, we further found that MMP7 was a direct target of miR-2276 in GBM cells. Since MMP7 is known to be highly involved in gliomagenesis, ${ }^{25}$ we focused on the regulation of MMP7 by miR-2276 and BCAR4. The results confirmed that MMP7 was downregulated by miR-2276 whereas upregulated by BCAR4 in GBM cells, demonstrating the existence of the BCAR4/miR-2276/MMP7 axis in glioma. Importantly, MMP7 restoration effectively rescued the impaired GBM cell growth and invasion caused by BCAR4 knockdown. Till now, we proved that MMP7 was an essential downstream effector of BCAR4 in promoting gliomagenesis.

In summary, our results provide solid evidences into the oncogenic roles of BCAR4 via targeting miR-2276 to upregulate MMP7 in gliomagenesis. These findings may improve the understanding of the glioma pathogenesis and improve the clinical prognosis and therapy for the patients.

\section{Ethical Approval and Written Informed Consent}

All procedures performed in this study involving human participants were in accordance with the 1964 Helsinki declaration. Written informed consent was obtained from all patients enrolled in this study.

\section{Disclosure}

The authors report no conflicts of interest in this work.

\section{References}

1. Bray F, Ferlay J, Soerjomataram I, Siegel RL, Torre LA, Jemal A. Global cancer statistics 2018: GLOBOCAN estimates of incidence and mortality worldwide for 36 cancers in 185 countries. CA Cancer J Clin. 2018;68(6):394-424. doi:10.3322/caac.v68.6

2. Wen PY, Kesari S. Malignant gliomas in adults. $N$ Engl $J$ Med. 2008;359(5):492-507. doi:10.1056/NEJMra0708126

3. Clarke J, Butowski N, Chang S. Recent advances in therapy for glioblastoma. Arch Neurol. 2010;67(3):279-283. doi:10.1001/ archneurol.2010.5

4. Tykocki T, Eltayeb M. Ten-year survival in glioblastoma. A systematic review. J Clin Neurosci. 2018;54:7-13. doi:10.1016/j. jocn.2018.05.002

5. Chan JJ, Tay Y. Noncoding RNA:RNA regulatory networks in cancer. Int J Mol Sci. 2018;19(5):E1310. doi:10.3390/ijms19051 310

6. Peng S, Cao L, He S, et al. An overview of long noncoding RNAs involved in bone regeneration from mesenchymal stem cells. Stem Cells Int. 2018;2018:8273648. doi:10.1155/2018/8273648

7. Tang Q, Hann SS. HOTAIR: an oncogenic long non-coding RNA in human cancer. Cell Physiol Biochem. 2018;47(3):893-913. doi:10.1159/ 000490131

8. Wu Y, Shao A, Wang L, et al. The role of lncRNAs in the distant metastasis of breast cancer. Front Oncol. 2019;9:407. doi:10.3389/ fonc. 2019.00407

9. Lu T, Wang Y, Chen D, Liu J, Jiao W. Potential clinical application of lncRNAs in non-small cell lung cancer. Onco Targets Ther. 2018;13 (11):8045-8052. doi:10.2147/OTT.S178431

10. Li ZX, Zhu QN, Zhang HB, Hu Y, Wang G, Zhu YS. MALAT1: a potential biomarker in cancer. Cancer Manag Res. 2018;10:6757-6768. doi:10.2147/CMAR.S169406

11. Li J, Zhu Y, Wang H, Ji X. Targeting long noncoding RNA in glioma: a pathway perspective. Mol Ther Nucleic Acids. 2018;13:431-441. doi:10.1016/j.omtn.2018.09.023

12. Sen R, Ghosal S, Das S, Balti S, Chakrabarti J. Competing endogenous RNA: the key to posttranscriptional regulation. ScientificWorldJournal. 2014;2014:896206. doi:10.1155/2014/896206

13. Ergun S, Oztuzcu S. Oncocers: ceRNA-mediated cross-talk by sponging miRNAs in oncogenic pathways. Tumour Biol. 2015;36 (5):3129-3136. doi:10.1007/s13277-015-3346-x

14. Yang $\mathrm{C}, \mathrm{Wu} \mathrm{D}, \mathrm{Gao} \mathrm{L}$, et al. Competing endogenous RNA networks in human cancer: hypothesis, validation, and perspectives. Oncotarget. 2016;7(12):13479-13490. doi:10.18632/oncotarget.72 66

15. Huang J, Lyu H, Wang J, Liu B. MicroRNA regulation and therapeutic targeting of survivin in cancer. Am J Cancer Res. 2014;5 (1):20-31.

16. Qu J, Li M, Zhong W, Hu C. Competing endogenous RNA in cancer: a new pattern of gene expression regulation. Int J Clin Exp Med. 2015;8(10):17110-17116.

17. Shuwen H, Qing Z, Yan Z, Xi Y. Competitive endogenous RNA in colorectal cancer: a systematic review. Gene. 2018;645:157-162. doi:10.1016/j.gene.2017.12.036

18. Ju L, Zhou YM, Yang GS. Up-regulation of long non-coding RNA BCAR4 predicts a poor prognosis in patients with osteosarcoma, and promotes cell invasion and metastasis. Eur Rev Med Pharmacol Sci. 2016;20(21):4445-4451.

19. Zou R, Chen X, Jin X, et al. Up-regulated BCAR4 contributes to proliferation and migration of cervical cancer cells. Surg Oncol. 2018;27(2):306-313. doi:10.1016/j.suronc.2018.05.013 
20. Ouyang S, Zhou X, Chen Z, Wang M, Zheng X, Xie M. LncRNA BCAR4, targeting to miR-665/STAT3 signaling, maintains cancer stem cells stemness and promotes tumorigenicity in colorectal cancer. Cancer Cell Int. 2019;19:72. doi:10.1186/s12935-019-0784-3

21. Wei L, Yi Z, Guo K, Long X. Long noncoding RNA BCAR4 promotes glioma cell proliferation via EGFR/PI3K/AKT signaling pathway. $J$ Cell Physiol. 2019;234:23608-23617. doi:10.1002/jcp.28929

22. Shui X, Zhou C, Lin W, Yu Y, Feng Y, Kong J. Long non-coding RNA BCAR4 promotes chondrosarcoma cell proliferation and migration through activation of mTOR signaling pathway. Exp Biol Med (Maywood). 2017;242(10):1044-1050. doi:10.1177/1535370217700735

23. Yang H, Yan L, Sun K, et al. IncRNA BCAR4 increases viability, invasion, and migration of non-small cell lung cancer cells by targeting glioma-associated oncogene 2 (GLI2). Oncol Res. 2019;27 (3):359-369. doi:10.3727/096504018X15220594629967

24. Paraskevopoulou MD, Vlachos IS, Karagkouni D, et al. DIANALncBase v2: indexing microRNA targets on non-coding transcripts. Nucleic Acids Res. 2016;44(D1):D231-D238. doi:10.1093/nar/gkv1270
25. Mu N, Gu J, Liu N, et al. PRL-3 is a potential glioblastoma prognostic marker and promotes glioblastoma progression by enhancing MMP7 through the ERK and JNK pathways. Theranostics. 2018;8 (6):1527-1539. doi:10.7150/thno.22699

26. Peng $\mathrm{Z}$, Liu $\mathrm{C}, \mathrm{Wu} \mathrm{M}$. New insights into long noncoding RNAs and their roles in glioma. Mol Cancer. 2018;17(1):61. doi:10.1186/ s12943-018-0812-2

27. Tan SK, Pastori C, Penas C, et al. Serum long noncoding RNA HOTAIR as a novel diagnostic and prognostic biomarker in glioblastoma multiforme. Mol Cancer. 2018;17(1):74. doi:10.1186/s12943018-0822-0

28. Torkashvand S, Damavandi Z, Mirzaei B, Tavallaei M, Vasei M, Mowla SJ. Decreased expression of bioinformatically predicted piwil2-targeting microRNAs, miR-1267 and miR-2276 in breast cancer. Arch Iran Med. 2016;19(6):420-425.

\section{Publish your work in this journal}

OncoTargets and Therapy is an international, peer-reviewed, open access journal focusing on the pathological basis of all cancers, potential targets for therapy and treatment protocols employed to improve the management of cancer patients. The journal also focuses on the impact of management programs and new therapeutic agents and protocols on patient perspectives such as quality of life, adherence and satisfaction. The manuscript management system is completely online and includes a very quick and fair peer-review system, which is all easy to use. Visit http://www.dovepress.com/ testimonials.php to read real quotes from published authors. 